\title{
Dipolar energy states in clusters of perpendicular magnetic nanoislands
}

\author{
E. Mengotti, ${ }^{1}$ L. J. Heyderman, ${ }^{1, a)}$ A. Bisig, ${ }^{1}$ A. Fraile Rodríguez, ${ }^{1}$ L. Le Guyader, ${ }^{1}$ \\ F. Nolting, ${ }^{1}$ and $\mathrm{H}$. B. Braun ${ }^{2}$ \\ ${ }^{1}$ Paul Scherrer Institut, 5232 Villigen PSI, Switzerland \\ ${ }^{2}$ School of Physics, University College Dublin, Belfield, National University of Ireland, Dublin 4, Ireland
}

(Received 30 March 2009; accepted 13 April 2009; published online 4 June 2009)

\begin{abstract}
We investigated the energy states in compact clusters of ferromagnetic islands with perpendicular anisotropy arranged on a triangular lattice. Due to their finite nature, we were able to determine the energies of all possible cluster states using dipolar energy calculations. We employed photoemission electron microscopy to observe the magnetic states in arrays of clusters of monodomain $\mathrm{Co} / \mathrm{Pt}$ multilayer islands and following demagnetization, we observed a shift in the energy distribution to lower energies as the dipolar coupling increased. These multistate island clusters not only provide model arrangements of frustrated Ising-type nanomagnets but are also interesting for data storage applications. () 2009 American Institute of Physics. [DOI: 10.1063/1.3133202]
\end{abstract}

With their unique magnetic fingerprint, clusters of regularly spaced magnetic islands with perpendicular anisotropy are interesting for data storage applications. In particular, for magnetic recording the interest in bit patterned media, which consists of arrays of perpendicular magnetic islands, arises from the need to find ways to increase the current magnetic recording densities by reducing the bit size while staying above the superparamagnetic limit. ${ }^{1,2} \mathrm{~A}$ bit of information is recorded in a single island and the highest density of recorded information is given by arrays of islands arranged on a triangular (or hexagonal) lattice. In order to write to a single island, the magnetic fields produced by the recording head must be large enough to switch the island but also local enough to avoid unintended switching of neighboring islands. As an alternative, a "cluster media" consisting of arrays of identical clusters of magnetic islands could be implemented. Here, the information would be written to the whole cluster using, for example, a spatially larger field with multilevel field strength capabilities to access the different magnetic states. In order to apply such a scheme, it is important as a first step to identify the available magnetic states and their energies, and gain an idea of the accessibility of these states. In addition, such clusters of perpendicular islands on a triangular lattice provide fundamental model systems to directly observe frustrated behavior - a phenomenon present in many physical systems ${ }^{3,4}$ - since, as we will show later, for three neighboring islands the magnetic dipolar interactions between pairs of islands can never all be satisfied at once. Indeed, the clusters bear some similarities to the classical triangular Ising antiferromagnet. ${ }^{5}$ However, in contrast to the Ising antiferromagnet where only the nearest-neighbor exchange interactions are taken into account, long range dipolar interactions beyond the nearest neighbors are important.

In this paper we explore different cluster arrangements of perpendicular nanoislands on a triangular lattice. We determine the energies of all of the possible states using a dipolar calculation. Employing photoemission electron micros-

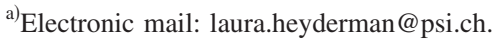

copy (PEEM), we directly observe the magnetic states in arrays of island clusters following demagnetization with an ac magnetic field. We find that there is an increase in the frequency of low energy states as the coupling strength increases. Due to its simplicity and the easy accessibility of the various states, the three-island cluster shows promise for future data storage.

We focus our study on compact clusters consisting of two to seven islands, choosing island arrangements on a triangular lattice, which minimize the total area covered by the island cluster [see Fig. 1]. We first calculate the dipolar energy for all of the $2^{N}$ possible configurations for each cluster, where $N$ is the number of islands (details of the calculation can be found in Ref. 4). The full set of energy plots for all of the clusters with $N=2-7$ are given in the supplemental

\begin{tabular}{|c|c|c|c|}
\hline $\mathrm{N}$ & $\begin{array}{l}\text { Ground } \\
\text { state }\end{array}$ & $\begin{array}{l}\text { Number } \\
\text { of states }\end{array}$ & $\begin{array}{c}\text { Number of } \\
\text { energy levels }\end{array}$ \\
\hline 2 & 0 & 4 & 2 \\
\hline 3 & & 8 & 2 \\
\hline 4 & & 16 & 5 \\
\hline 5 & & 32 & 9 \\
\hline 6 & & 64 & 9 \\
\hline 7 & & 128 & 13 \\
\hline
\end{tabular}

FIG. 1. Geometries of the different clusters of perpendicular islands on a triangular lattice with the number of islands, $N=2-7$. One of the degenerate ground state configurations is shown for each cluster together with its degree of degeneracy, the total number of possible states $\left(2^{N}\right)$, and the number of energy levels obtained from the energy calculation. ${ }^{6}$ For the different magnetic states, the islands with moments pointing up (down) are white (black), and the favorable (unfavorable) interactions are given by the dashed (continuous) line. 
information. ${ }^{6}$ For each plot, the states with the same energy are grouped together in levels and ordered with increasing energy from left to right. In general, for each cluster, an increase in dipolar energy is associated with an increase in the number of unfavorable island-island interactions and/or an increase in the total magnetization $\left(M_{\uparrow}-M_{\downarrow}\right)$.

From the energy calculations, the lowest energy ground state configurations can be determined and are given in Fig. 1 together with their degree of degeneracy. In addition, the number of possible states $\left(2^{N}\right)$ and the number of energy levels are indicated. The magnetic moment of each island can point either up or down (white or black, corresponding to moments pointing out of or into the surface plane), and for neighboring islands it is energetically favorable to have one island with the moment pointing up and one island with the moment pointing down. This up-down configuration is indeed the ground state for the two-island cluster, which has a twofold degeneracy and no frustration is present. On increasing the number of islands, frustration is introduced and is immediately apparent in the ground state of the basic threeisland cluster in Fig. 1, where one island-island interaction is always unfavorable with parallel island moments indicated by the continuous line. The other two interactions have favorable up-down (white-black) island interactions, indicated by a dashed line between the islands. In order to describe this moment configuration, we introduce the expression that the three neighboring islands "obey the three-island rule" (i.e., with a one-up/two-down moment configuration or vice versa). For the four-island cluster, the ground state consists of two three-island subclusters obeying the three-island rule, which are combined in such a way that the unfavorable interaction (given by the continuous line) is shared by the subclusters. In the five-island cluster, the ground state consists of the four-island cluster ground state with one additional island leading to a pair of unfavorable interactions parallel to each other. The ground state of seven-island cluster also contains several three-island subclusters, which all obey the threeisland rule, and the unfavorable interactions are shared between the subclusters. All of the clusters described so far are made up of three-island clusters obeying the three-island rule and have the same ground state configurations predicted for a finite triangular Ising antiferromagnet. ${ }^{5}$ However, for the sixisland cluster, there is one central three-island subcluster [three central black dots in Fig. 1] which does not obey the three-island rule and deviates from the ground state configuration predicted for the triangular Ising antiferromagnet, ${ }^{5}$ which involves nearest neighbor exchange rather than long range dipolar interactions.

We next try to observe experimentally whether it is possible to access the lowest energy states in a real system. For this, we produced arrays of $6 \times 6$ identical island clusters of $\mathrm{Co} / \mathrm{Pt}$ multilayer islands with electron beam lithography. ${ }^{4}$ The multilayer films of $\mathrm{Pt}(1.8 \mathrm{~nm}) /[\mathrm{Co}(0.5 \mathrm{~nm}) /$ $\operatorname{Pt}(1.8 \mathrm{~nm})]_{6}$ were deposited with dc magnetron sputtering. The islands have a diameter $d=410 \mathrm{~nm}$ and we took two values of the interisland spacing corresponding to a lattice parameter [see Fig. 2(a)], $p=510 \mathrm{~nm}$ (high dipolar coupling) and $p=810 \mathrm{~nm}$ (low dipolar coupling). Neighboring clusters have sufficient separation $(>2 p)$ to avoid intercluster dipolar
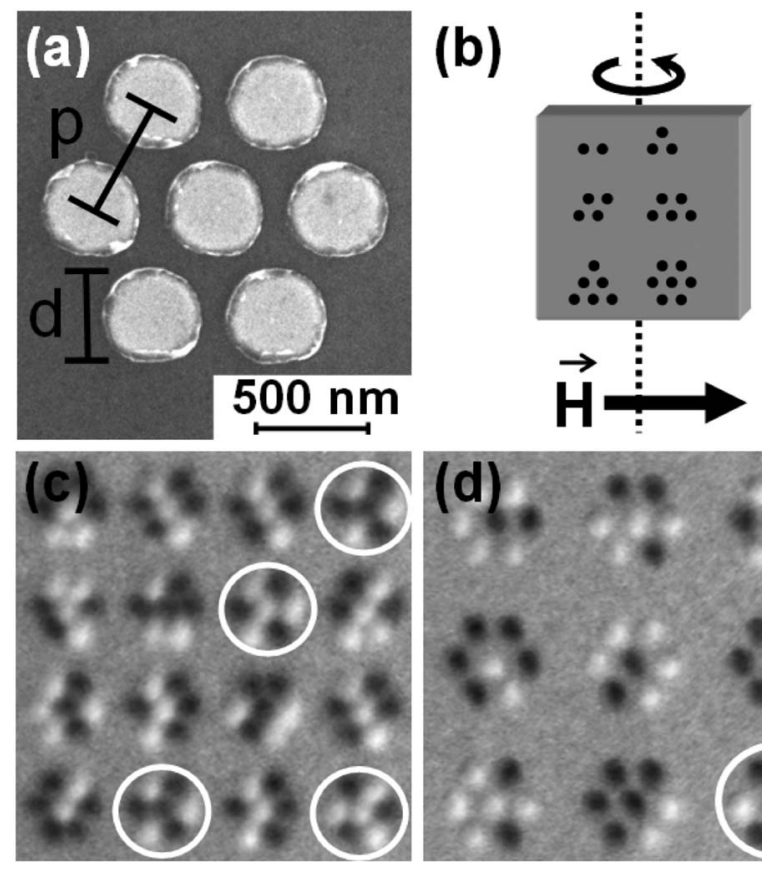

up down

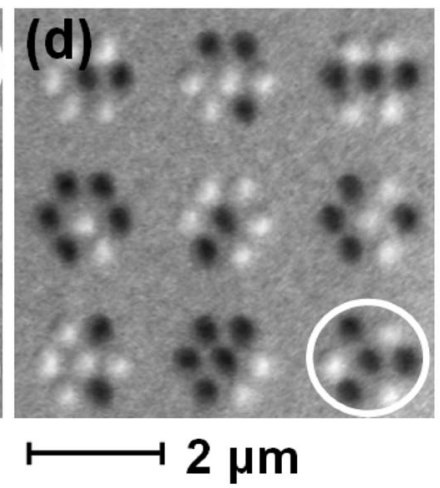

FIG. 2. (a) Scanning electron microscope image of the seven-island cluster with diameter, $d=410 \mathrm{~nm}$ and lattice parameter $p=510 \mathrm{~nm}$. (b) Schematic of the demagnetization setup with the sample rotating about an in-plane axis. XMCD images of the seven-island cluster following demagnetization with $d=410 \mathrm{~nm}$, and (c) $p=510 \mathrm{~nm}$ and (d) $p=810 \mathrm{~nm}$. The uniform contrast associated with each island indicates that they are monodomain with the magnetic moments pointing perpendicular to the sample plane, either up (white) or down (black). The ground states are highlighted with a circular frame.

coupling. We demagnetized a sample containing all of the different arrays of clusters by rotating the sample about an in-plane axis [see demagnetization geometry in Fig. 2(b)] at a frequency of $2 \mathrm{~Hz}$ in a magnetic field, starting with a field value of $740 \mathrm{mT}$, well above the magnetization saturation value, and reducing it down to zero at approximately $5 \mathrm{mT} / \mathrm{s}$. We then observed the magnetic states with PEEM at the Swiss Light Source (SIM beamline), taking advantage of the $\mathrm{x}$-ray magnetic circular dichroism (XMCD) effect, ${ }^{7}$ which with our setup is sensitive to the out-of-plane magnetization component. XMCD images of part of the array of sevenisland clusters for both the small and the large lattice parameters are shown in Figs. 2(c) and 2(d). The islands have a uniform white (black) contrast corresponding to islands with uniform moments pointing up (down), indicating that the macrospin approximation used for the dipolar energy calculations holds. While most of the three-island subclusters obey the three-island rule, there is a small number of threeisland subclusters which do not: $4 \%$ at high coupling and $12 \%$ at low coupling ( $25 \%$ is expected at zero coupling). The islands do not show a preferred orientation with zero net moment in the sample.

We determine the frequency of states following demagnetization of the entire set of island cluster arrays [Figs. 3(a)-3(f)], and compare these values with the random frequencies expected for zero interisland dipolar coupling. The random frequency is simply given by the degree of degen- 

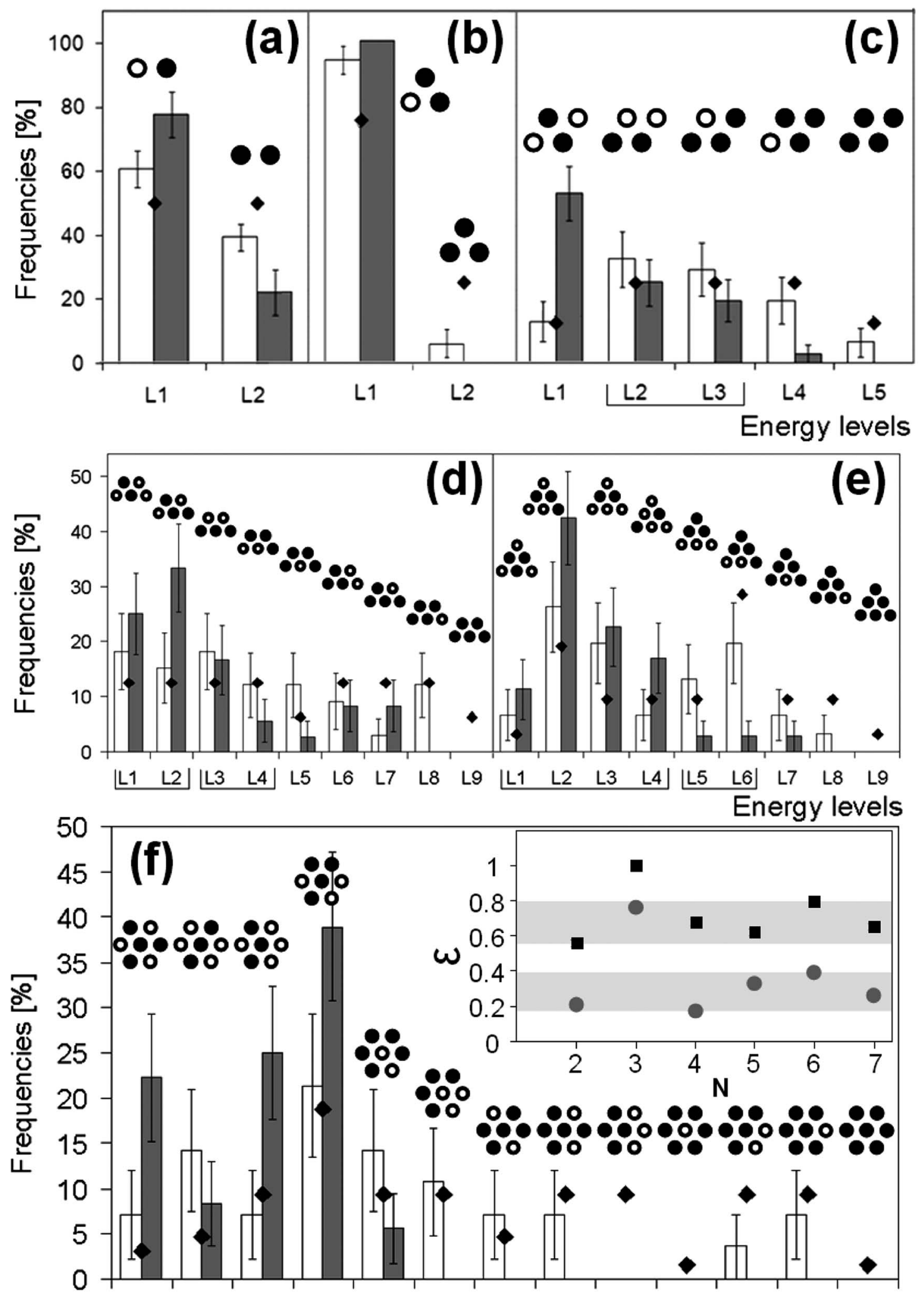

L1 LL2 L3

Energy levels

FIG. 3. Observed frequency of states for the different island clusters, including the random distribution expected for zero interisland coupling (rhombuses) and the experimentally measured values for low coupling ( $p=810 \mathrm{~nm}$, white bars) and high coupling ( $p=510 \mathrm{~nm}$, gray bars). For each level, one of the possible magnetic configurations is shown and levels which are close in energy ${ }^{6}$ are grouped in brackets. In the inset, the values of the demagnetization effectiveness parameter, $\varepsilon$, for all clusters, divided into clusters with high coupling (squares) and low coupling (circles), are given. 
eracy of each state divided by the total number of states. To interpret the data, we introduce a demagnetization effectiveness parameter $\varepsilon$ which we define as $\varepsilon=\langle E\rangle / E_{\mathrm{GS}}$, where $\langle E\rangle$ is the average dipolar energy per configuration observed on demagnetization and $E_{\mathrm{GS}}$ is the ground state energy of the given $N$-island cluster. When $\varepsilon=0$, the distribution of energy states would be purely random (which gives $\langle E\rangle=0$ ) and when $\varepsilon=1$, all of the clusters in the array would be in the ground state (i.e., $\langle E\rangle=E_{\mathrm{GS}}$ ). The $\varepsilon$ values for all clusters, separated into clusters with high coupling (squares) and low coupling (circles), are plotted in the inset of Fig. 3(f). Excluding for the moment the data for the three-island cluster, all other clusters at low coupling show a frequency distribution close to the random distribution with $\varepsilon$ in the range $0.17-0.39$. This implies that any residual coupling between the dots does not play a significant role in determining the acquired states. This can also be seen in the detailed frequency plots [Figs. 3(a) and 3(c)-3(f)] where the random distribution (rhombuses) is more or less mimicked by the measured values (white bars). At high coupling, $\varepsilon$ increases to be in the range of $0.56-0.79$, indicating that the energy distribution moves to lower energies. However, since $\varepsilon<1$, there is still evidence of the random distribution. Focusing on the seven-island cluster [Fig. 3(f)], we see that for high interisland dipolar coupling (gray bars), the levels above L5 are no longer occupied and there is a sharp increase in the number of ground states. For the remaining levels there is still a peak in the frequency values at L4, mirroring the peak in the random distribution but having a higher value due to the occupancy of fewer levels. This general behavior is seen in all of the other clusters [Figs. 3(a) and 3(c)-3(e)], although in the five- and six-island clusters there appears to be a relatively low number of ground states, which is likely to be due to the close proximity of the next highest energy levels ${ }^{6}$ as indicated by the brackets under the plots. The effectiveness parameter for the three-island cluster is astonishingly high. At low coupling, $\varepsilon=0.76$, and at high coupling, all 36 threeisland clusters fall into the ground state giving $\varepsilon=1$. Therefore, it seems that the ground states can be captured with an ac magnetic field with a high reliability irrespective of the coupling strengths employed here. This can be partly explained by the fact that in the random limit, it is three times more likely to achieve the ground state with its sixfold degeneracy (L1) than the higher energy state (L2), which has a twofold degeneracy.

In conclusion we have investigated the behavior of clusters of perpendicular islands on a frustrated triangular lattice. Employing dipolar energy calculations, we are able to deter- mine the energies of all of the possible states and therefore identify the lower energy states. Experimentally we observed the frequencies of states as a function of lattice parameter following demagnetization with an ac magnetic field. We observed that there is a shift to lower energy states as the dipolar coupling is increased. While we are not able to bring the islands much closer together without them touching, a further shift in the energy distribution, brought about by a higher interisland dipolar coupling, could be attained by increasing the moment of the magnetic material. The presence of these well-defined energy levels may be of interest for future data storage, not only in computer hard drives but also for information tags, ${ }^{8}$ replacing the recording of a barcode. Implementation in a solid state device, such as magnetoresistive random access memory, would reduce the number of contact leads required. For data storage the cluster size should be optimized to have a large enough number of the available states while minimizing the complexity. The threeisland cluster, with its simplicity and eight magnetic states, four of which are accessible with either a dc or an ac magnetic field, would be a good candidate. A key task for the future will be to determine avenues for achieving specific cluster states reproducibly in real systems.

The authors would like to thank Michael Horisberger for the sputter deposition, Anja Weber, Eugen Deckardt, and Christian David for their support with electron beam lithography, as well as Andrea Steger for support at the beamline. This work was supported by the Swiss National Science Foundation and the Science Foundation of Ireland, and part of this work was performed at the Swiss Light Source, Paul Scherrer Institut, Villigen, Switzerland.

${ }^{1}$ T. Thomson, G. Hu, and B. D. Terris, Phys. Rev. Lett. 96, 257204 (2006). ${ }^{2}$ F. Luo, L. J. Heyderman, H. H. Solak, T. Thomson, and M. E. Best, Appl. Phys. Lett. 92, 102505 (2008).

${ }^{3}$ R. F. Wang, C. Nisoli, R. S. Freitas, J. Li, W. McConville, B. J. Cooley, M. S. Lund, N. Samarth, C. Leighton, V. H. Crespi, and P. Schiffer, Nature (London) 439, 303 (2006).

${ }^{4}$ E. Mengotti, L. J. Heyderman, A. Fraile Rodriguez, A. Bisig, L. Le Guyader, F. Nolting, and H. B. Braun, Phys. Rev. B 78, 144402 (2008).

${ }^{5}$ R. P. Millane, A. Goyal, and R. C. Penney, Phys. Lett. A 311, 347 (2003).

${ }^{6}$ See EPAPS Document No. E-JAPIAU-105-123910 for the energy plots of compact clusters of perpendicular islands on a triangular lattice with $N=2$ to 7 . For more information on EPAPS, see http://www.aip.org/ pubservs/epaps.html.

${ }^{7}$ J. Stohr, H. A. Padmore, S. Anders, T. Stammler, and M. R. Scheinfein, Surf. Rev. Lett. 5, 1297 (1998).

${ }^{8}$ J.-R. Jeong, J. Llandro, B. Hong, T. J. Hayward, T. Mitrelias, K. P. Kopper, T. Trypiniotis, S. J. Steinmuller, G. K. Simpson, and J. A. C. Bland, Lab Chip 8, 1883 (2008). 